\title{
The Aeolus Data Innovation and Science Cluster (DISC)
}

\section{Isabell Krisch ${ }^{1}$}

Oliver Reitebuch ${ }^{1}$, Jonas von Bismarck², Tommaso Parrinello², Michael Rennie ${ }^{3}$, Fabian Weiler ${ }^{1}$, Dorit Huber ${ }^{4}$, Jos de Kloe ${ }^{5}$, Alain Dabas ${ }^{6}$, Anne Grete Straume-Lindner ${ }^{7}$, Saleh Abdalla ${ }^{3}$, Stefano Aprile ${ }^{2}$, Sebastian Bley ${ }^{8}$, Fabio Bracci ${ }^{9}$, Simone Bucci ${ }^{10}$, Massimo Cardaci $^{10}$, Werner Damman ${ }^{11}$, Dave Donovan $^{5}$, Frithjof Ehlers ${ }^{7}$, Frederic Fabre ${ }^{12}$, Peggy Fischer ${ }^{2}$, Thomas Flament ${ }^{6}$, Alexander Geiß ${ }^{13}$, Phil McGoldrick ${ }^{12}$, Giacomo Gostinicchi ${ }^{10}$, Lars Isaksen ${ }^{3}$, Sebastian Jupin-Langlois ${ }^{14}$, Thomas Kanitz ${ }^{7}$, Adrien Lacour ${ }^{6}$, Marta De Laurentis ${ }^{2}$, Christian Lemmerz ${ }^{1}$, Oliver Lux ${ }^{1}$, Uwe Marksteiner ${ }^{1}$, Gert-Jan Marseille ${ }^{5}$, Nafiseh Masoumzadeh ${ }^{1}$, Markus Meringer ${ }^{9}$, Sander Niemeijer ${ }^{11}$, Ines Nikolaus ${ }^{15}$, Gaetan Perron ${ }^{14}$, Bas Pijnacker-Hordijk ${ }^{11}$, Katja Reissigi ${ }^{16}$, Matic Savli ${ }^{6}$, Karsten Schmidt ${ }^{9}$, Ad Stoffelen $^{5}$, Dimitri Trapon $^{6}$, Michael Vaughan ${ }^{17}$, Marcella Veneziani ${ }^{11}$, Cristiano De Vincenti ${ }^{10}$, Benjamin Witschas ${ }^{1}$

${ }^{1}$ DLR, Institute of Atmospheric Physics ${ }^{2} E S A-E S R I N$

${ }^{3}$ ECMWF

${ }^{4}$ DoRIT

${ }^{5} \mathrm{KNMI}$

${ }^{6}$ Météo-France

${ }^{7}$ ESA-ESTEC

${ }^{8}$ TROPOS
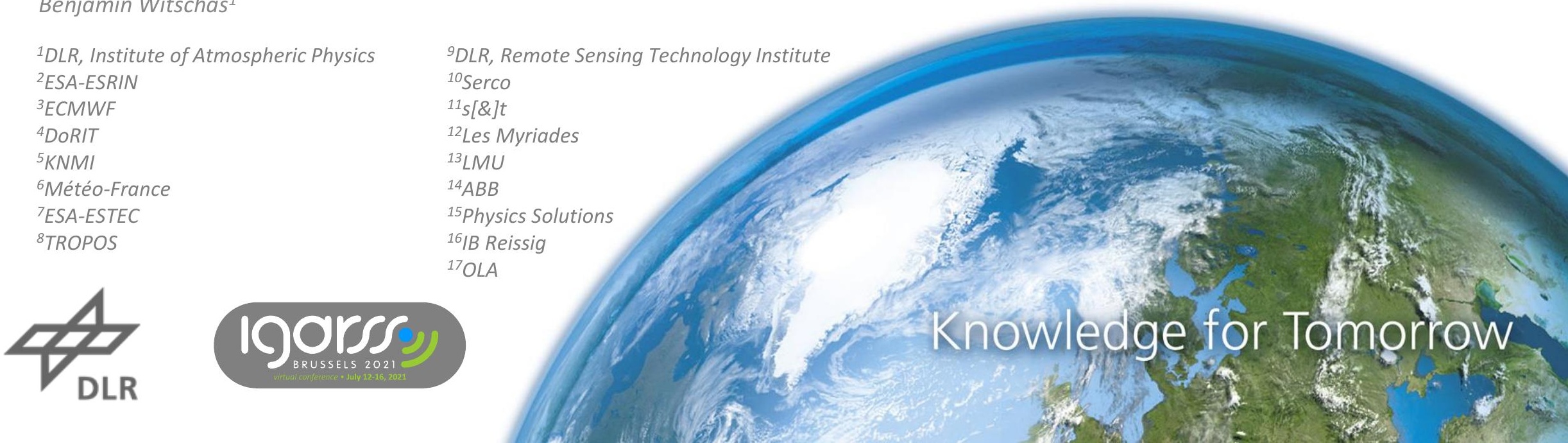

19orsse

${ }^{10}$ Serco

${ }^{11} s[\&] t$

${ }^{15}$ Physics Solutions

${ }^{16}$ IB Reissig

${ }^{17}$ OLA 


\section{The Aeolus Data Innovation and Science Cluster (DISC)}

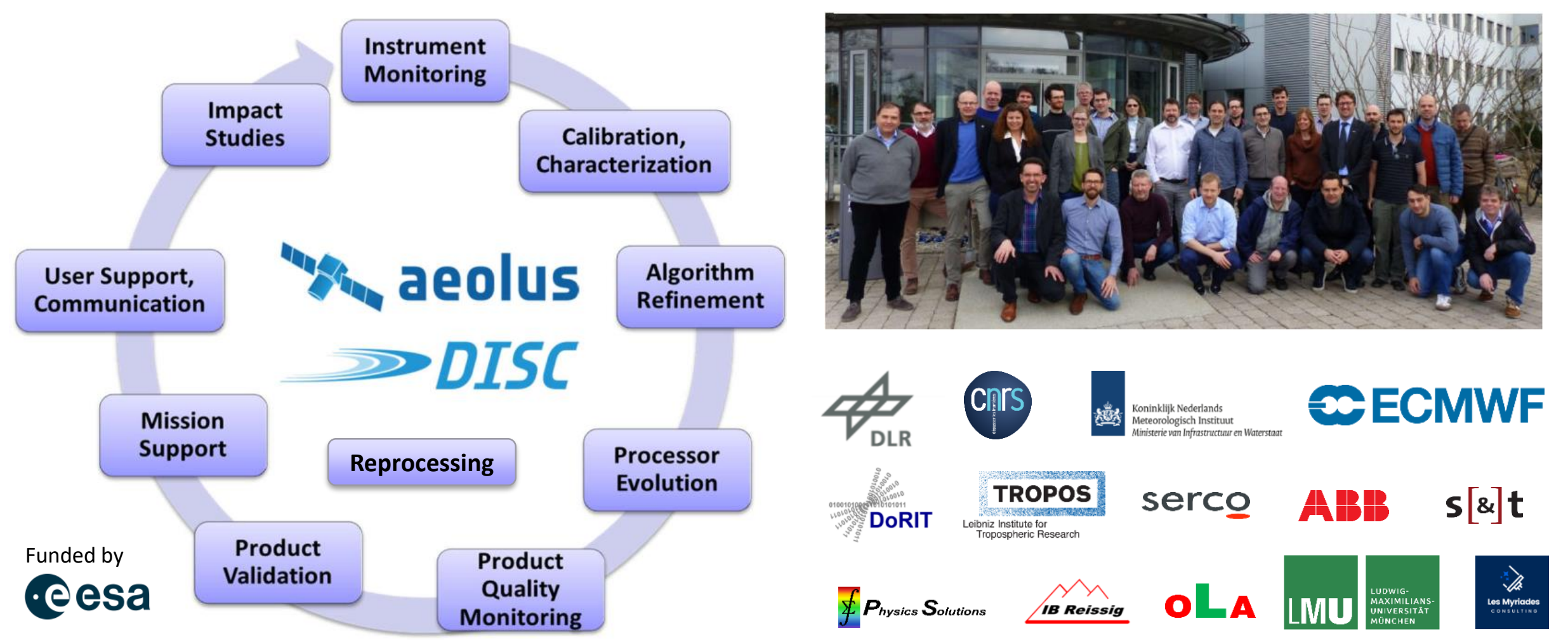




\section{Operational monitoring of Aeolus near-real-time data at ECMWF}
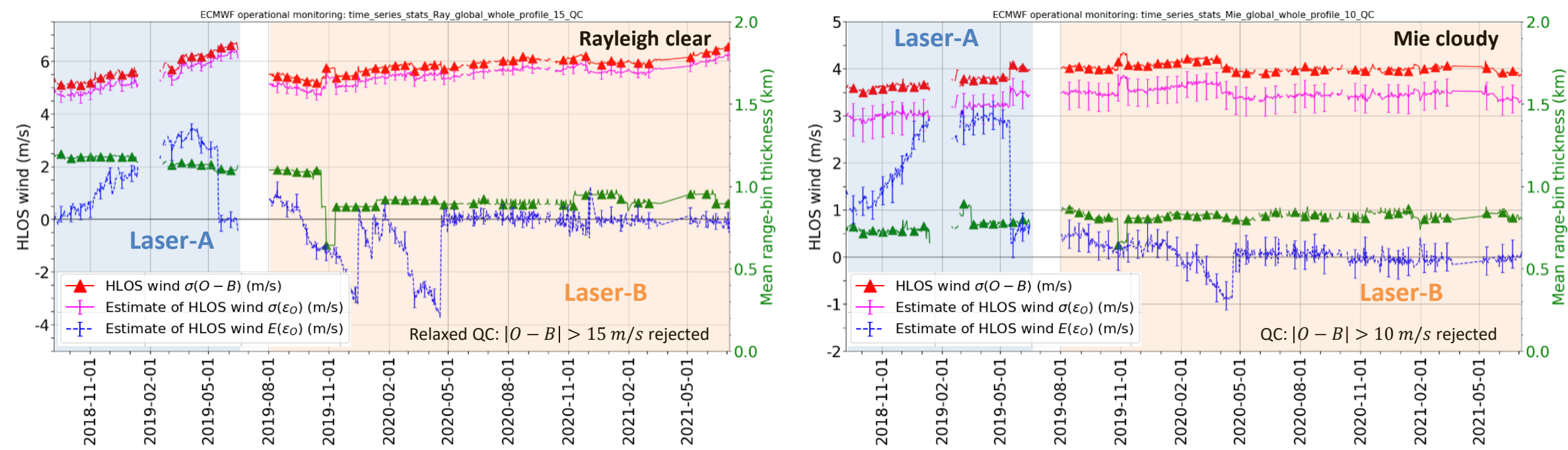

- Aeolus is the first satellite mission to implement operational monitoring at ECMWF directly after launch.

Figures by M. Rennie (ECMWF). This operational monitoring is a very strong tool and helped to identify several before launch unexpected error sources.

- After launch, the systematic errors (bias) for both Mie and Rayleigh winds (several $\mathrm{m} / \mathrm{s}$ ) showed strong slow drifts, orbital variations, differences for ascending and descending orbits, and stronger biases in single range-gates.

Since 20 April 2020 global mean bias for both channels is around $0 \mathrm{~m} / \mathrm{s}$

- The Aeolus random error is currently in the order of $6 \mathrm{~m} / \mathrm{s}$ for Rayleigh winds and $3.5 \mathrm{~m} / \mathrm{s}$ for Mie winds
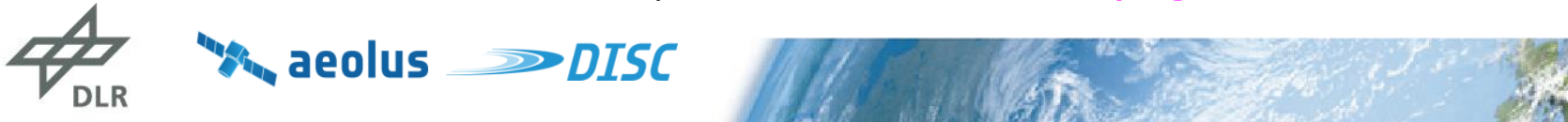


\section{What causes systematic errors?}

\section{Combination of several unexpected error sources}

with different temporal characteristics

* Thermal variations of the M1 telescope mirror

$\Rightarrow$ Corrected with Baseline 09 (20 April 2020)

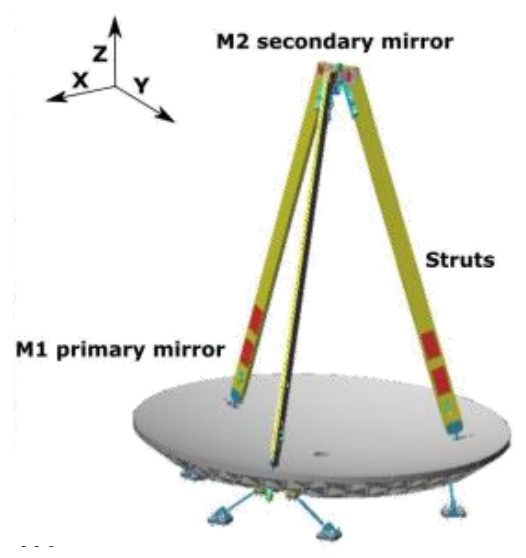

Thermal variations of M1 telescope mirror depend on outgoing longwave radiation
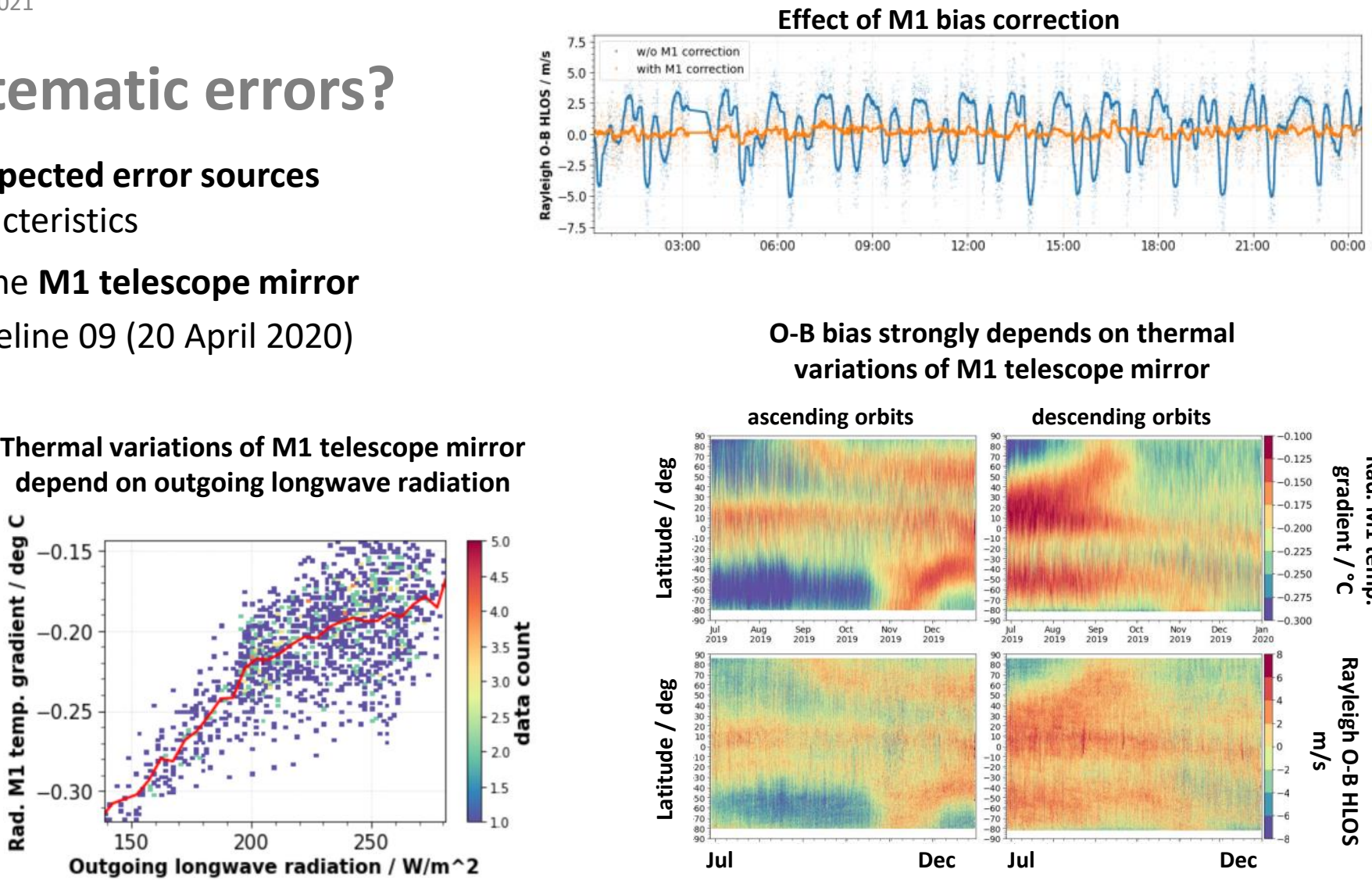

O-B bias strongly depends on thermal variations of $\mathrm{M} 1$ telescope mirror

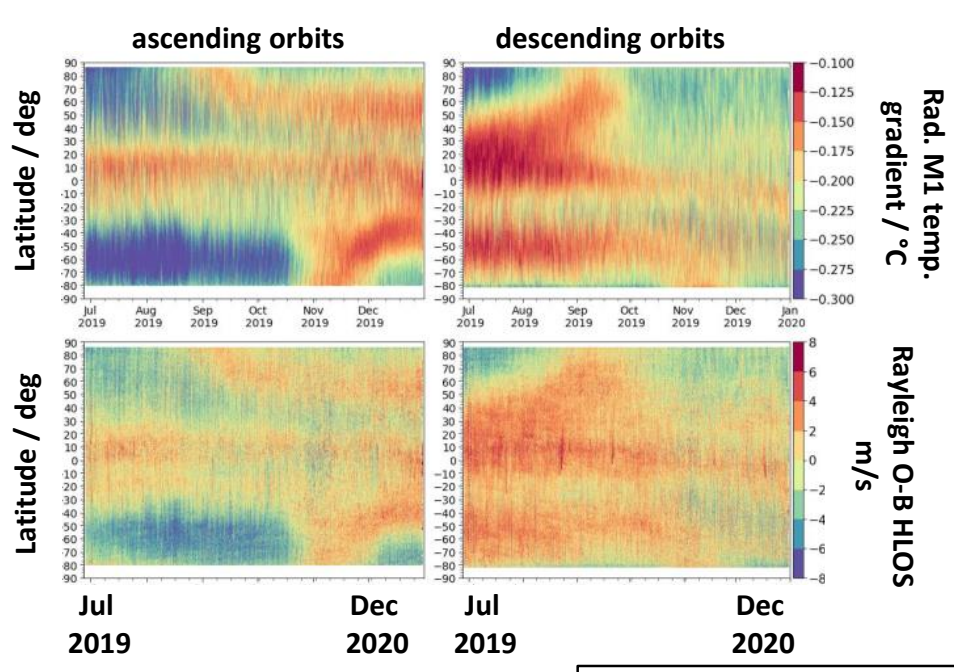

All figures from Weiler et al., AMTD 2021 


\section{What drives the random errors?}

\section{* Laser emit energy}

$\Rightarrow$ Lower than expected (factor 1-2)

$\Rightarrow$ Negative trend

* Optical signal throughput in receive path for atmospheric signal

$\Rightarrow$ Lower than expected (factor 2-3)

$\Rightarrow$ Negative trend

\section{* Solar background noise}

$\Rightarrow$ Impact higher than expected due to lower atmospheric signal

$\Rightarrow$ Seasonal variation of solar background by factor 18: Rayleigh random errors of 7-8 m/s were obtained in summer months for polar regions

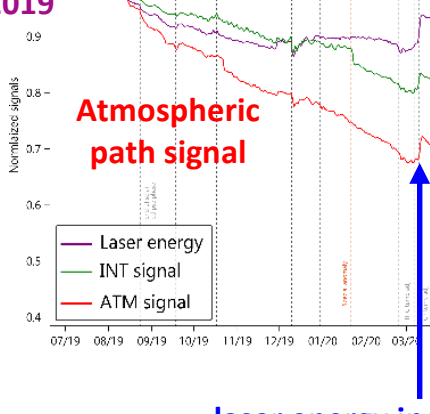

laser energy increase in March and December 2020

Figure by $\mathbf{O}$. Lux (DLR).

\section{Seasonal variation of Rayleigh solar background noise}

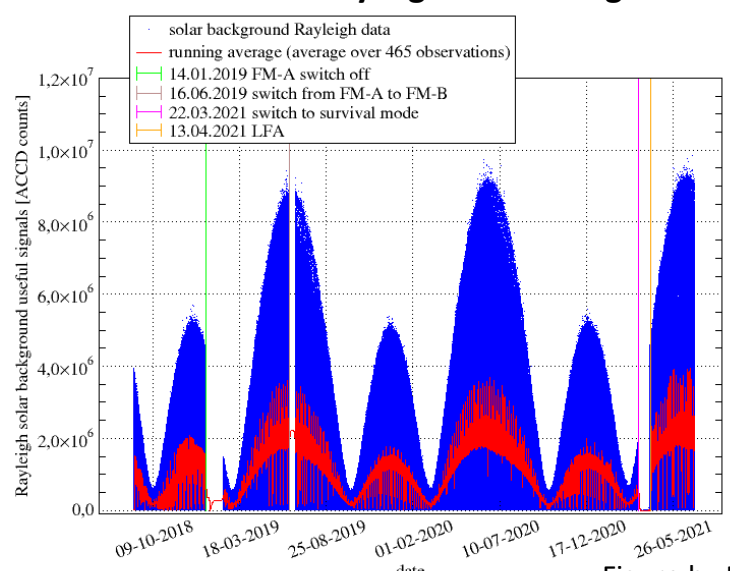

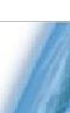




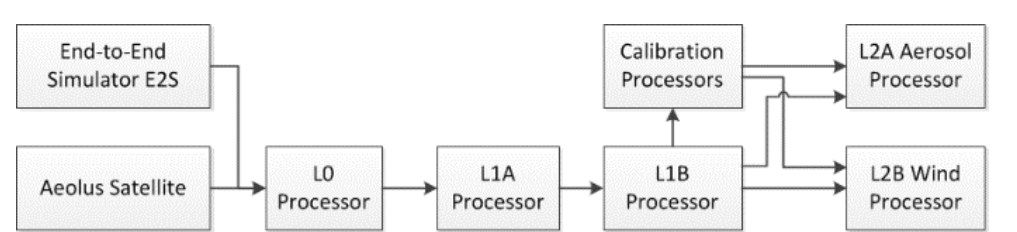

- New processor versions from DISC and baseline update for NRT and reprocessing every 6 months with improvements in data quality for all products.

- Current focus is the further development of the L2A processor and products. Baseline 12 products (since May 2021) e.g. include lidar ratios and a scene heterogeneity index.

- Additionally, a new feature mask (based on EarthCare algorithms) is available since baseline 12 (beta version!) and a new optimal estimation retrieval for backscatter and extinction will be added with baseline 13 (autumn 2021).

- Recently, a new correction for the Mie-cloudy winds was introduced, which significantly reduced the Mie systematic and random errors.

- We are currently working on a Rayleigh-clear "altitude dependent" bias correction for the L2B wind products.

Aeolus Feature Mask in L2A product

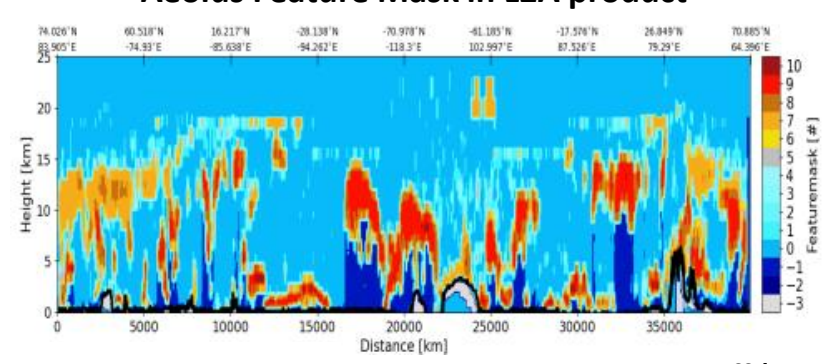

Figure by G-J. v. Zadelhoff (KNMI).

"Altitude dependent" Rayleigh bias

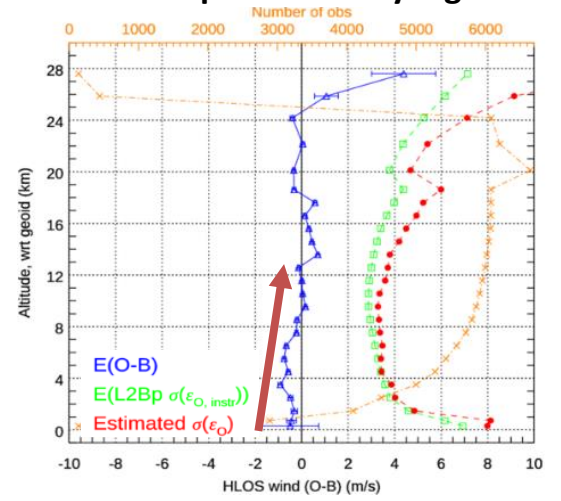

Figure by M. Rennie (ECMWF). 


\section{Reprocessing of Aeolus data}

- $\quad 1^{\text {st }}$ reprocessing from June to December 2019 with baseline 10 product quality finished and available since October 2020.

- $\quad 2^{\text {nd }}$ re-processing campaign is on-going using baseline 11 processor versions (L1B 7.09, L2A 3.11, L2B 3.40) for period June 2019 to October 2020

- fill gap in bias correction from Jan-May 2020 and consistent processing for 15 months of data

- calibration of L2A product with varying $\mathrm{K}_{\text {ray }}$ and $\mathrm{K}_{\text {mie }}$ along the orbit

- small improvements in bias correction for hot-pixel and M1

- relaxed ground detection thresholds -> more ground returns available

- $\quad$ Plans for 2022:

- Reprocessing of laser-A data with baseline 13

- Reprocessing of complete Aeolus mission with baseline 14

Re-processed laser-B data of 2019

including M1 correction available on

14 October 2020

Oct 2020

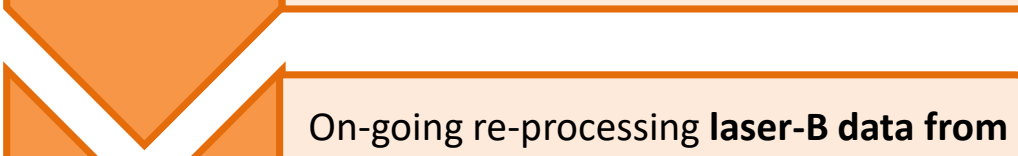

June 2019 to October 2020 will become available in autumn 2021

autumn 2021

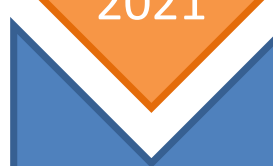

early 2022

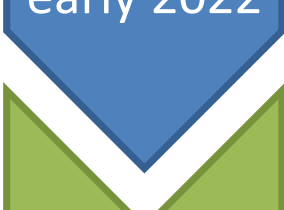

End 2022
Re-processed laser-A data with hot-pixel and M1 correction will become available in early 2022
Re-processing of complete Aeolus mission since launch with optimized processor settings and all available corrections. 


\section{Summary and Conclusion}

- The Aeolus DISC consortium is responsible for instrument monitoring, calibration, processor evolution, product quality, user support and impact studies.

- Aeolus wind data is monitored at ECMWF since launch.

- This concept allowed a fast detection and correction of multiple systematic biases. Since 20 April 2020 global mean bias for both channels (Rayleigh \& Mie) is around $0 \mathrm{~m} / \mathrm{s}$.

- The random error is larger than expected before launch and currently in the order of $6 \mathrm{~m} / \mathrm{s}$ for Rayleigh winds and $3.5 \mathrm{~m} / \mathrm{s}$ for Mie winds.

- New processor versions from DISC and baseline update for NRT and reprocessing are provided every 6 months with improvements in data quality for all products.

- First re-processed data (June - December 2019) available since Oct. 2020. More to come in autumn 2021.
Aeolus data quality is constantly monitored at ECMWF

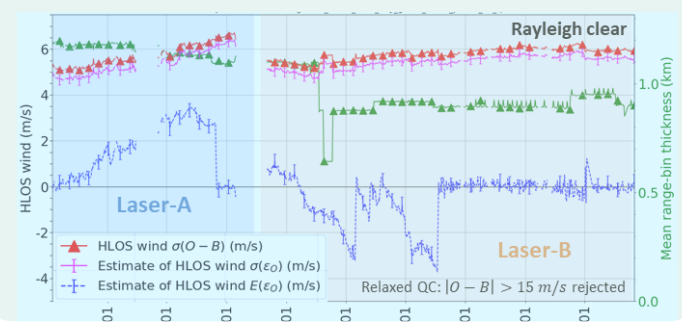

Several systematic errors have been corrected since launch

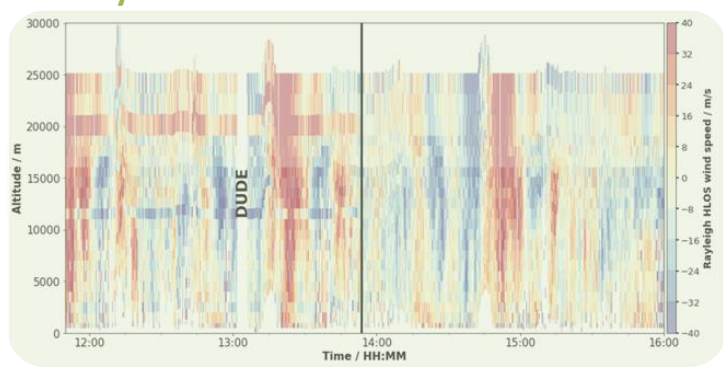

Reprocessing further enhances data quality

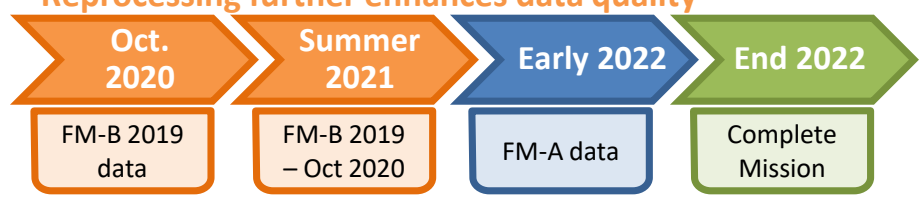

